\title{
Controlled trial of an Internet-based intervention for overweight teens (Next.Step): effectiveness analysis
}

\author{
Pedro Sousa • Helena Fonseca • Pedro Gaspar • \\ Filomena Gaspar
}

Received: 21 August 2014 /Revised: 30 January 2015 /Accepted: 10 February 2015 /Published online: 14 March 2015

(C) Springer-Verlag Berlin Heidelberg 2015

\begin{abstract}
Adolescent obesity is a major health problem. The need for effective adolescent weight management programs is of high clinical and public health relevance. This study evaluates the effectiveness of an e-therapeutic platform (Next.Step), aiming to promote weight management skills and the adoption of health-promoting lifestyles. This nonrandomized clinical trial with control group uses a sample of 94 adolescents who attended a paediatric obesity clinic. The experimental group was invited to access the platform Next.Step during 24 weeks, in addition to the standard treatment program. The control group followed the standard treatment protocol and joined a waiting list. Overall, the outcomes
\end{abstract}

\begin{tabular}{l} 
Communicated by Jaan Toelen \\
Revisions received: 20 December 2014 / 30 January 2015 \\
\hline Trial registration: NCT01904474 \\
\hline P. Sousa ( $\square) \cdot$ P. Gaspar \\
School of Health Sciences, Polytechnic Institute of Leiria, \\
Leiria, Portugal \\
e-mail: pedro.sousa@ipleiria.pt \\
P. Gaspar \\
e-mail: pedrgaspar@gmail.com \\
P. Sousa • P. Gaspar \\
Health Research Unit (UIS) of the Polytechnic Institute of Leiria, \\
Leiria, Portugal \\
H. Fonseca \\
Faculdade de Medicina da Universidade de Lisboa, Lisbon, Portugal \\
e-mail: helenaregalofonseca@gmail.com \\
H. Fonseca \\
Paediatric Obesity Clinic, Department of Paediatrics, Hospital de \\
Santa Maria (HSM), Lisbon, Portugal \\
F. Gaspar \\
Escola Superior de Enfermagem de Lisboa, Lisbon, Portugal \\
e-mail: mfgaspar@esel.pt
\end{tabular}

of the e-therapeutic program were not substantially better than those obtained with a traditional kind of intervention despite the intervention being associated with an improved health responsibility score $(d=0.51 ; p=0.014)$. Several predictors of the Next.Step effectiveness were found.

Conclusion: Although the e-therapeutic program led to a significant increase in health responsibility, inconclusive results were found regarding the program effectiveness compared to the standard multidisciplinary intervention. The lack of significant differences between groups may be due to the reduced rates of program adherence and the high dropout rate.

What is Known:

- Adolescent obesity has reached epidemic proportions, and standard treatment programs have been unable to achieve the desired adherence so far.

- Internet-based programs have already shown positive results in the adult obese population, but information is scarce regarding adolescents.

What is New:

- This study assesses the effectiveness of a structured case management program including communication technologies on both the behavioural change and the health of obese adolescents.

- The e-therapeutic program led to an increase in health responsibility.

Keywords Teen obesity · Adolescence - Overweight · Case management $\cdot$ Internet-based $\cdot$ Weight management

$\begin{array}{ll}\text { Abbreviations } \\ \text { ALP } & \text { Adolescent lifestyle profile } \\ \text { AWCQ } & \text { Adherence to Weight Control Questionnaire } \\ \text { BE } & \text { Body esteem } \\ \text { BMI } & \text { Body mass index } \\ \text { CDC } & \text { Centers for Disease Control and Prevention } \\ \text { FR } & \text { Family relations } \\ \text { FSI } & \text { Friends and school influence }\end{array}$




$\begin{array}{ll}\text { h/w } & \text { hours/week } \\ \text { HR } & \text { Health responsibility } \\ \text { IR } & \text { Interpersonal relations } \\ \text { ITT } & \text { Intention-to-treat analysis } \\ \text { IWQOL } & \text { Impact of Weight on Quality of Life } \\ \text { N } & \text { Nutrition } \\ \text { NSUQ } & \text { Next.Step Usability Questionnaire } \\ \text { PA } & \text { Physical activity } \\ \text { PB } & \text { Perceived benefits } \\ \text { PC } & \text { Physical comfort } \\ \text { PLP } & \text { Positive life perspective } \\ \text { PPI } & \text { Parents and providers influence } \\ \text { RNAWC } & \text { Risk of non-adherence to weight control } \\ \text { SEA } & \text { Self-efficacy and adherence behaviours } \\ \text { SH } & \text { Spiritual health } \\ \text { SL } & \text { Social life } \\ \text { SM } & \text { Stress management } \\ \text { T0 } & \text { Baseline evaluation } \\ \text { T2 } & \text { Post-intervention evaluation } \\ \text { TAWC } & \text { Treatment adherence to weight control } \\ \text { WHO } & \text { World Health Organization }\end{array}$

\section{Introduction}

Adolescent obesity is a major health problem with an urgent need for effective interventions that induce behavioural change. Successful treatments depend on lifestyle change and on the maintenance of the therapeutic support [5]. According to the literature, ongoing contact between the patient and the health care provider leads to greater adherence to behavioural changes, resulting in improved maintenance of weight lost. The Internet has established itself as a viable and promising option in this field $[1,2,8,10]$.

Recent systematic reviews $[4,26]$ on the effectiveness of information and communication technologies (ICT) in the treatment and prevention of obesity in children and adolescents concluded that most intervention programs produced significant effectiveness in reducing BMI, fat intake and increasing physical activity, supporting the promise and potential of Internet-based interventions as a viable component of weight management programs.

In 2011, we conducted a systematic literature review on the impact of e-therapeutic interventions in the treatment of obese adolescents [34]. The results supported the potential of this kind of interventions as a vital component of weight management programs and stressed the need for additional studies in order to optimize the Internet as an effective communication channel for teenagers.

Baulch, Chester and Brennan [9] further stressed the importance of differentiating between individuals likely to benefit from an online program and those for whom a face-to-face program should be more appropriate. Making this differentiation a priori can be particularly relevant since the online interventions are not universally appealing.

This study seeks to expand on these results, particularly focusing on the inclusion of the management case methodology in the e-therapy programs and on the predictors of the effectiveness of intervention programs. The primary objective of this study was to determine the effectiveness of the intervention program compared to the conventional treatment, in what concerns the behaviour changes (promotion of therapeutic adherence and healthy lifestyles) and the impact on the adolescent's health of the weight control. Empirical evidence was collected for the general hypothesis that therapeutic adherence, healthy lifestyles, quality of life and weight control in obese adolescents were positively influenced by the intervention program. The secondary objective was to explore the predictors of the effectiveness of the e-therapeutic intervention program.

\section{Materials and methods}

Study design

This study is designed as a nonrandomized clinical trial (quasi-experimental pre- and post-test design with a control group). We are looking for empirical evidence for the general investigation hypothesis: treatment adherence, healthy lifestyle, quality of life and weight control of obese adolescents are positively influenced by this intervention program.

\section{Participants}

Participants were obese adolescents from a paediatric obesity clinic (Portugal), aged between 12 and 18 years (BMI percentile $\geq 95$ th), with Internet access of at least once a week (inclusion criteria). Exclusion criteria were the presence of severe psychopathology, inability to communicate in writing, pregnancy or having been proposed for bariatric surgery. The sample recruitment had the support of the clinical staff.

All eligible adolescents with appointments between 2012 January 1 and December 31 were included in the study. The participants were consecutively allocated into the experimental and control groups according to the sample size calculation. Sample size calculation was conducted as described previously in the study protocol [33], assuming the need for showing differences between groups with a standardized effect size of 0.4 or larger, a $30 \%$ retention rate, an alpha of 0.05 and a statistical power (1-Beta) of $80 \%$. Therefore, there was a need for recruiting at least 75 adolescents. To maximize the statistical power of the study design, we have expanded the sample to 94 adolescents ( 48 in the experimental group and 46 in the control arm). 
The experimental group was divided into two subgroups, depending on the study enrolment order: group $\mathrm{A}(n=23)$ and group B $(n=25)$. Group A began the intervention in May 2012 and group B in October 2012, aiming to reduce the adolescents' waiting period for starting the programme. The procedure was identical in both groups, namely, the treatment length, methods, content/resources and functionalities, case manager and clinical team.

Experimental group participants, in addition to the standard treatment program consisting of clinical evaluation and medical, psychological, nutritional and physical activity counselling, were invited to get restricted access to the e-therapeutic platform (Next.Step).

The control group followed the standard clinical treatment protocol, which includes individual visits to the paediatrician, dietician and exercise physiologist. These adolescents joined a waiting list to access Next.Step in the short run.

\section{Ethical considerations}

This study was approved by the Ethical Committee for Health and funded by the Foundation for Science and Technology (PTDC/DTP-PIC/0769/2012) in 2012. All procedures respected the Declaration of Helsinki [43] and the ethical principles of the American Psychological Association [3]. All eligible adolescents and respective parents signed the informed consent where the study objectives were explained, following the ethical aspects of confidentiality and voluntary participation.

\section{Measures}

Program effectiveness was evaluated every 3 months, with emphasis at baseline evaluation (T0), intermediate evaluation (12 weeks-T1) and post-intervention assessment (24 weeks - T2). The data were obtained through three main sources: (a) clinical file (demographics, anthropometric, behavioural and clinical variables), (b) self-report instruments (AWCQ_Adherence to Weight Control Questionnaire, ALP_Adolescent Lifestyle Profile and IWQOL_-Impact of Weight on Quality of Life), (c) Next.Step e-therapeutic platform (utilisation rate, logins, consulted resources, self-monitoring data, game scores, participation in forums). The instruments used are described in detail in the study protocol [33].

BMI assessment Anthropometric data were measured by trained health professionals from the clinic. For assessing platform effectiveness, WHO BMI charts [27] were used in addition to the CDC BMI charts [21] to enable comparison of results with studies using either approach.

Behavioral variables These include weekly physical activity $(\mathrm{h} / \mathrm{w})$, screen time $(\mathrm{h} / \mathrm{w})$, family support and weight loss motivation (two Likert-type questions, range 1-5) and body image (sequence of seven silhouettes that evolve progressively from thinness to overweight).

Usability assessment NSUQ included a user profile evaluation section (internet access, amount of internet daily use, computer availability) and a second section regarding the evaluation of the usability perception (27 independent items, scored 1-5; the higher the index, the better the usability).

Adherence to Weight Control Questionnaire (AWCQ) This instrument was developed and validated by Sousa, Fonseca and Gaspar [31]. This screening tool measures treatment adherence to weight control (TAWC) and the risk of non-adherence to weight control (RNAWC) in adolescents, with a five-point Likert-type format. The TAWC (29 items) includes four subscales: SEA (self-efficacy and adherence behaviours), PPI (parental and providers influence), FSI (friends and school influence) and PB (perceived benefits). The RNAWC (seven items) presented a one-factor solution. Both scales presented good reliability values $(0.908$ and 0.770$)$ and a five-point Likert-type format. A high TAWC score corresponds to a greater treatment adherence. Furthermore, a high RNAWC score corresponds to a greater risk of nonadherence.

Adolescent Lifestyle Profile (ALP) This instrument was designed to measure the frequency of health-promoting behaviours in adolescents (early, middle and late). The Portuguese version of ALP [32] is a 36-item summated behaviour rating scale that employs a four-point Likert-type response format, organized into seven factors (health responsibility, physical activity, nutrition, positive life perspective, interpersonal relationship, stress management and spiritual health). The scale has a high reliability score $(\alpha=0.866)$, with subscale reliability values between 0.492 and 0.747 . A high ALP score corresponds to a healthier lifestyle.

Impact of Weight on Quality of Life (IWQOL) This questionnaire consists of 27 items organized into four factors (physical comfort, body esteem, social life and family relations). For the purpose of this study, we used the Portuguese version [28].

Intervention

Participants were invited to get restricted access to the etherapeutic platform (Next.Step), which included a diverse set of resources, such as educational resources (videos, brochures, menus, weekly tips, access to other links), selfmonitoring (food, weight and physical activity records), social support (chats, discussion forums and personalized 
messages), interactive training modules (self-assessment quizzes, making their own diets) and motivational tools (personal goals planning, treatment progression registry, positive reinforcement). In addition to the platform manager (nurse), the program also had the direct support of an interdisciplinary team (including paediatrician, nutritionist, exercise physiologist and psychologist) who intervened when requested by the case manager. The intervention length was 24 weeks, being based on case management methodology. The program was structured in ten thematic modules, with an average duration of 2 weeks. The modular structure of Next.Step seeks to create a virtual environment attractive to adolescents, according to the logic of a computer game, where the player must accomplish specific tasks/missions before progressing to the following level, unlocking new features and functionalities. Full presentation of the platform features can be found in detail in the study protocol [33].

\section{Analysis}

Data were analysed with the Statistical Package for Social Sciences (SPSS v.17) using nonparametric tests since sample data had no normal distribution. Descriptive statistics were calculated, and the groups were compared regarding their initial characteristics (T0-baseline assessment) by MannWhitney $U$ test $(U)$ for continuous variables and the chisquare test $\left(\chi^{2}\right)$ for nominal variables. In order to compare the effect of the two intervention programs (standard program and Next.Step program), data were analysed using generalized linear models with the likelihood ratio test $(\Lambda)$. The final assessment of the dependent variables (T2) was included in these models, with the group as fixed factor and the baseline values (T0) as covariates.

The missing data were determined by the expectationmaximization method (EM, implemented by the Missing Value Analysis of the SPSS). The Little's MCAR test (Missing Completely At Random) confirmed the assumption of randomization of missing data since the differences were not statistically significant $(p>0.05)$.

An intention to treat analysis (ITT) and a parallel per protocol analysis - PP (considering only the adolescents who in fact accessed the e-therapeutic platform) were conducted. The effect sizes were calculated using the partial eta squared $\left(\eta^{2}\right)$ that indicates the proportion of the total variability attributable to a given factor. The standardized effect sizes (Cohen's $d$ ) would be further calculated in the presence of significant differences between the two intervention programs. The effect analysis of the interventions, within the same group (paired samples), was performed using the Wilcoxon-signed ranks test. All analyses were performed using bilateral tests, with a significance level of 0.05 .

The predictive effect of anthropometric, sociodemographic, behavioural and clinical data on the effectiveness of the e- therapeutic program was analysed at 24 weeks, using nonparametric tests (Spearman correlation, Mann-Whitney $U$ test and Kruskal-Wallis test).

\section{Results}

Analysis of adherence/attrition of the intervention program

In the experimental arm, 23 participants (47.92\%) were excluded, one for having been proposed for bariatric surgery (exclusion criterion) and 22 by having never accessed the platform (Fig. 1). Significant differences were found between the baseline characteristics of the experimental group participants who have adhered (adherents) and those who have abandoned the intervention program (nonadherents) regarding sedentary lifestyle (adherents, 18.87 \pm 9.83 ; non-adherents, $24.92 \pm 8.46 ; p=0.015$ ), nutrition (adherents, 2.91 \pm 0.41 ; non-adherents, $2.63 \pm 0.49$; $p=$ 0.042 ), influence of parents and health professionals in adherence to treatment (adherents, $4.68 \pm 0.35$; non-adherents, $4.36 \pm 0.51 ; p=0.024)$.

The adherents, on average, accessed the platform 10.68 times $(\mathrm{SD}=18.92)$ and on average analysed $7.87(\mathrm{SD}=9.25)$ resources and read $31.77(\mathrm{SD}=47.56)$ messages from the forums during the 24-week time period. The average number of reported data (weight, physical activity and nutrition) was $3.00(\mathrm{SD}=4.85)$, and the percentage of completed activities was $13.66 \%$ ( $\mathrm{SD}=47.56)$. Regarding parents, only one out of the 28 parents who joined the program accessed the platform (attrition rate of $96.43 \%$ ).

Analysis of baseline characteristics of participants

The mean age of the adolescents was 14.17 years $(\mathrm{SD}=1.51)$, with average BMI z-score of $2.66(\mathrm{SD}=0.72)$, according to WHO reference curves. The percentage of girls $(51.06 \%)$ was similar to that of boys (48.94 \%). The indices of weekly physical activity were $4.54 \pm 2.89 \mathrm{~h} / \mathrm{w}$, and the rates of sedentary lifestyle were $22.48 \pm 8.65 \mathrm{~h} / \mathrm{w}$. Results further showed indices of family support of $3.92 \pm 0.72$ and indices of personal motivation of $3.67 \pm 0.82$ (Table 1 ).

The average lifestyle profile global rate was $2.61(\mathrm{SD}=$ 0.38 ), the lowest result being found in the subscale of spiritual health and the highest result in the subscale of interpersonal relations. The average global rate of the weight-related quality of life was $79.90(\mathrm{SD}=18.90)$, with the lowest result in the subscale of body esteem and the highest result in the subscale of family relations.

The average global rate of adherence to weight control was $3.73(\mathrm{SD}=0.57)$, with an average risk of non-adherence of $2.51(\mathrm{SD}=0.86)$. 
Fig. 1 Study procedure

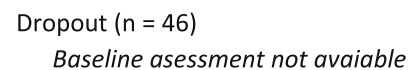

Dropout $(n=23)$

Non-adherence to Next.Step $(n=22)$ Waiting for bariatric surgery $(n=1)$

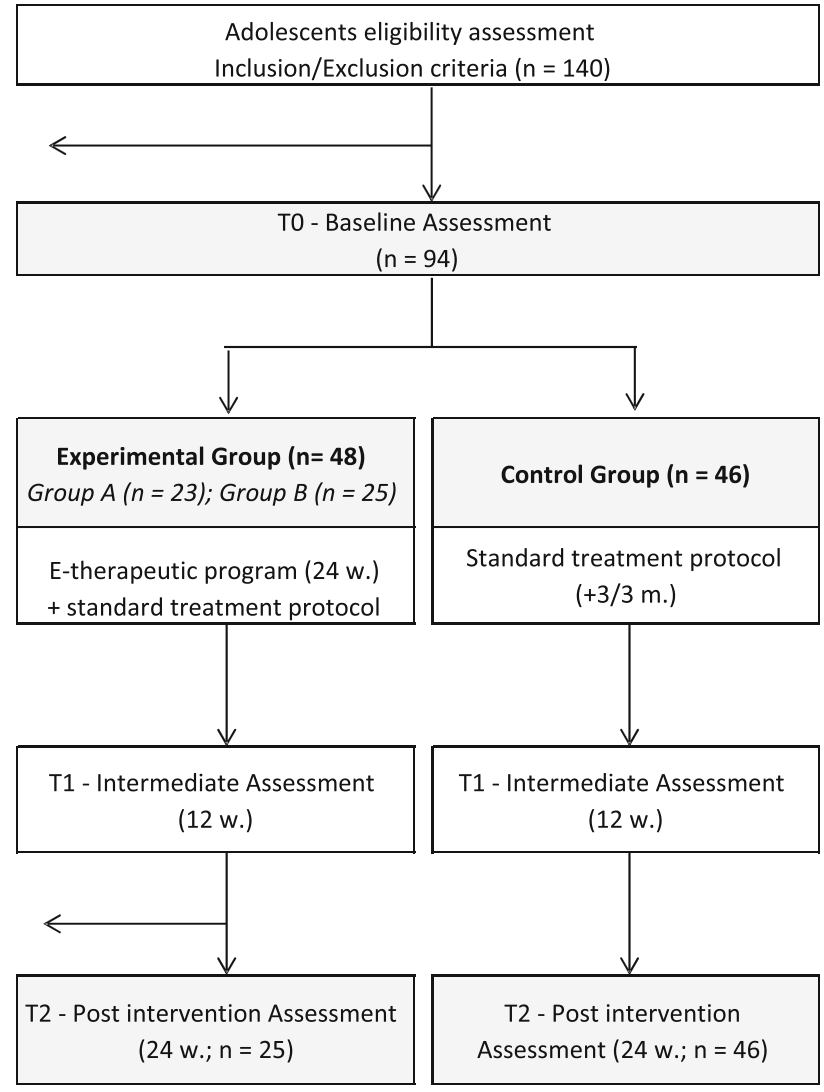

$0.113 ;=6.081 ; p=0.014)$ to the control group adolescents $(\Delta M=0.003 \pm 0.43)$ (Table 2). These differences correspond to a moderate effect size $(d=0.51)$ with clinical significance [12, 44].

Adherence to weight control The data analysis in T2 (postintervention evaluation) showed a significant reduction in the perception of adherence benefits, both for the control group ( $\Delta M=-0.07 \pm 0.33 ; Z=-2.017 ; p=0.044)$ and the experimental group (ITT and PP analysis: $\Delta M=-0.13 \pm 0.39 ; Z=-3.306$; $p=0.001$ vs $\Delta M=-0.24 \pm 0.29 ; Z=-3.465 ; p=0.001$, respectively) (Table 2). The ITT analysis allowed for further identification of the presence of significant differences between the initial and final evaluation of the overall adherence to weight control index in the experimental group adolescents $(\Delta M=$ $-0.08 \pm 0.44 ; Z=-1.979 ; p=0.048)$. The covariance analysis between groups did not demonstrate the existence of significant differences $(p>0.05)$.

Effectiveness analysis regarding the impact on the health

Weight-related quality of life Post-intervention assessment found an improvement in all quality of life indices $(p<0.05)$ in both groups (Table 2). The analysis of covariance between groups did not show the existence of significant differences $(p>0.05)$. 
Table 1 Baseline characteristics of the participants of the experimental and control group

\begin{tabular}{|c|c|c|c|c|c|c|}
\hline & \multicolumn{2}{|c|}{ Experimental group } & \multicolumn{2}{|c|}{ Control group } & \multirow[t]{2}{*}{$U$} & \multirow[t]{2}{*}{$p$} \\
\hline & $M$ & SD & $M$ & $\mathrm{SD}$ & & \\
\hline Age & 14.35 & 1.56 & 13.98 & 1.44 & 958.500 & 0.261 \\
\hline \multicolumn{7}{|l|}{ Anthropometric data } \\
\hline BMI z-score (CDC) & 2.06 & 0.33 & 2.09 & 0.42 & 1069.000 & 0.791 \\
\hline BMI z-score (WHO) & 2.61 & 0.63 & 2.71 & 0.81 & 1082.500 & 0.871 \\
\hline Waist circumference percentile & 91.43 & 2.95 & 92.05 & 3.02 & 1035.500 & 0.602 \\
\hline Hip circumference & 108.94 & 7.44 & 109.94 & 8.57 & 1089.500 & 0.910 \\
\hline \multicolumn{7}{|l|}{ Behavioural variables } \\
\hline Weekly physical activity (h/w) & 4.36 & 3.14 & 4.73 & 2.63 & 912.500 & 0.144 \\
\hline Screen time $(\mathrm{h} / \mathrm{w})$ & 21.77 & 9.60 & 23.22 & 7.57 & 1039.500 & 0.620 \\
\hline Family support & 3.87 & 0.74 & 3.97 & 0.71 & 1061.000 & 0.740 \\
\hline Weight loss motivation & 3.78 & 0.79 & 3.56 & 0.84 & 958.500 & 0.261 \\
\hline Body image silhouette & 5.42 & 0.69 & 5.65 & 0.73 & 853.000 & 0.049 \\
\hline \multicolumn{7}{|l|}{ Clinical variables } \\
\hline $\begin{array}{l}\text { Previous treatment length } \\
\text { (months) }\end{array}$ & 20.32 & 22.09 & 26.64 & 27.20 & 986.500 & 0.373 \\
\hline Age of onset of obesity & 6.58 & 3.31 & 6.35 & 2.76 & 1055.500 & 0.713 \\
\hline $\begin{array}{l}\text { Systolic blood pressure } \\
\text { percentile }\end{array}$ & 75.10 & 22.64 & 78.46 & 20.84 & 1002.000 & 0.438 \\
\hline $\begin{array}{l}\text { Diastolic blood pressure } \\
\text { percentile }\end{array}$ & 38.48 & 27.80 & 39.66 & 22.68 & 1065.000 & 0.768 \\
\hline \multicolumn{7}{|l|}{ Lifestyle } \\
\hline Health responsibility & 2.25 & 0.61 & 2.31 & 0.56 & 1025.000 & 0.549 \\
\hline Physical activity & 2.36 & 0.64 & 2.49 & 0.72 & 947.000 & 0.233 \\
\hline Nutrition & 2.78 & 0.46 & 2.80 & 0.52 & 1072.500 & 0.811 \\
\hline Positive life perspective & 3.17 & 0.62 & 2.98 & 0.60 & 908.000 & 0.135 \\
\hline Interpersonal relationship & 3.12 & 0.55 & 3.08 & 0.55 & 1064.000 & 0.760 \\
\hline Stress management & 2.95 & 0.48 & 2.94 & 0.60 & 1075.500 & 0.828 \\
\hline Spiritual health & 1.84 & 0.67 & 1.82 & 0.62 & 1092.000 & 0.927 \\
\hline ALP total score & 2.61 & 0.41 & 2.61 & 0.36 & 1097.000 & 0.958 \\
\hline \multicolumn{7}{|l|}{ Adherence to weight control } \\
\hline $\begin{array}{l}\text { Self-efficacy/adherence } \\
\text { behaviours }\end{array}$ & 3.23 & 0.74 & 3.23 & 0.91 & 1091.000 & 0.922 \\
\hline Parents and providers influence & 4.53 & 0.46 & 4.42 & 0.69 & 1085.500 & 0.887 \\
\hline Friends and school influence & 3.50 & 0.78 & 3.47 & 0.83 & 1073.000 & 0.814 \\
\hline Perceived benefits & 4.34 & 0.79 & 4.15 & 0.95 & 995.500 & 0.402 \\
\hline TAWC total score & 3.76 & 0.51 & 3.71 & 0.64 & 1058.000 & 0.728 \\
\hline Risk of non-adherence & 2.61 & 0.91 & 2.40 & 0.79 & 970.000 & 0.310 \\
\hline \multicolumn{7}{|l|}{ Obesity-related quality of life } \\
\hline Physical comfort & 83.47 & 19.37 & 81.72 & 22.53 & 1099.500 & 0.973 \\
\hline Body esteem & 69.56 & 25.06 & 63.20 & 30.52 & 989.000 & 0.384 \\
\hline Social life & 86.77 & 18.21 & 79.71 & 24.96 & 909.000 & 0.133 \\
\hline Family relations & 94.92 & 10.82 & 92.75 & 20.57 & 1085.500 & 0.873 \\
\hline \multirow[t]{2}{*}{ IWQOL total score } & 82.11 & 15.48 & 77.59 & 21.84 & 1002.500 & 0.442 \\
\hline & Number, $N$ & Percent, \% & Number, $N$ & Percent, \% & $\chi^{2}$ & $p$ \\
\hline \multicolumn{7}{|l|}{ Gender } \\
\hline Male & 24 & 50.00 & 22 & 47.83 & 0.044 & 0.833 \\
\hline Female & 24 & 50.00 & 24 & 52.17 & & \\
\hline
\end{tabular}


Table 1 (continued)

\begin{tabular}{|c|c|c|c|c|c|c|}
\hline \multicolumn{7}{|l|}{ Mother's education } \\
\hline 1 st-3rd cycle & 27 & 65.85 & 24 & 61.54 & \multirow[t]{2}{*}{0.161} & \multirow[t]{2}{*}{0.688} \\
\hline Secondary-higher education & 14 & 34.15 & 15 & 38.46 & & \\
\hline \multicolumn{7}{|l|}{ Father's education } \\
\hline $1 \mathrm{st}-3 \mathrm{rd}$ cycle & 26 & 65.00 & 26 & 68.42 & \multirow[t]{2}{*}{0.103} & \multirow[t]{2}{*}{0.749} \\
\hline Secondary-higher education & 14 & 35.00 & 12 & 31.58 & & \\
\hline \multicolumn{7}{|l|}{ Mother's occupational status } \\
\hline Differentiated professions & 24 & 64.86 & 24 & 70.59 & \multirow[t]{2}{*}{0.265} & \multirow[t]{2}{*}{0.607} \\
\hline Poorly differentiated professions & 13 & 35.14 & 10 & 29.41 & & \\
\hline \multicolumn{7}{|l|}{ Father's occupational status } \\
\hline Differentiated professions & 22 & 61.11 & 23 & 69.70 & \multirow[t]{2}{*}{0.560} & \multirow[t]{2}{*}{0.454} \\
\hline $\begin{array}{l}\text { Poorly differentiated } \\
\text { professions }\end{array}$ & 14 & 38.89 & 10 & 30.30 & & \\
\hline
\end{tabular}

$A L P$ adolescent lifestyle profile, TAWC treatment adherence to weight control, IWQOL Impact of Weight on Quality of Life, $U$ Mann-Whitney $U$ test statistic

Overweight and clinical results At 24 weeks (T2), the results of the ITT and PP analysis point to a significant reduction of the BMI z-score $(p<0.05)$, waist circumference $(p<0.05)$, screen time $(p<0.05)$ and family support, with an increase of the hip circumference $(p<0.05)$. The comparative analysis of covariance between the groups did not allow for the identification of statistically significant differences $(p<0.05)$ (Table 3).

Analysis of the predictors of the experimental program effectiveness

Predictors of overweight reduction Adolescents with a higher weight loss motivation tended to show a significant decrease in the BMI z-score, both considering the CDC $\left(r_{\mathrm{s}}=-0.469 ; p<0.05\right)$ and the WHO criteria $\left(r_{\mathrm{s}}=-0.411\right.$; $p<0.05$ ) (Table 4).

Predictors of effectiveness regarding lifestyle Adolescents whose parents have higher occupational status tended to show a significant increase in the rates of physical activity $(\Delta M=$ $0.32 \pm 0.48 v s \Delta M=-0.14 \pm 0.60 ; U=31.000 ; p<0.05)$ and positive perspective towards life $(\Delta M=0.13 \pm 0.45$ vs $\Delta M=-0.35$ $\pm 0.41 ; U=26.500 ; p<0.05)$. More inactive adolescents tended to achieve poorer results in health responsibility $\left(r_{\mathrm{s}}=-0.471\right.$; $p<0.05)$, physical activity $\left(r_{\mathrm{s}}=-0.556 ; p<0.05\right)$, and overall lifestyle index $\left(r_{\mathrm{s}}=-0.440 ; p<0.05\right)$. The adolescents who perceive their body silhouette as more obese tended to get better results in health responsibility $\left(r_{\mathrm{s}}=0.440 ; p<0.05\right)$ and stress management $\left(r_{\mathrm{s}}=0.478 ; p<0.05\right)$. A significant negative correlation was seen between the age of onset of obesity and the results in the spiritual health domain $\left(r_{\mathrm{s}}=-0.562\right.$; $p<0.05)$. The adolescents who did not refer previous attempts to control weight showed a better evolution in the subscale of nutrition $(\Delta M=0.35 \pm 0.24$ vs $\Delta M=-0.07 \pm 0.39 ; U=10.500$; $p<0.05$ ) (Table 4).
Predictors of effectiveness regarding the adherence to weight control The adolescents with a greater initial waist circumference percentile tend to have lower results in the risk of non-adherence to weight control $\left(r_{\mathrm{s}}=-0.423\right.$; $p<0.05)$. The adolescents whose mothers have lower education showed poorer results in perceiving the benefits of treatment adherence $(\Delta M=-0.29 \pm 0.20$ vs $\Delta M=$ $-0.13 \pm 0.35 ; U=29.500 ; p<0.05)$. Regarding the predictor effect of behavioural variables, it was possible to verify the existence of a negative correlation between the family support and the results obtained in the subscale of self-efficacy/adherence behaviours $\left(r_{\mathrm{s}}=-0.388\right.$; $p<0.05)$. A higher personal motivation for weight loss was associated to a lower influence of friends and school $\left(r_{\mathrm{s}}=-0.449 ; p<0.05\right)$ and to a decrease of the overall index of treatment adherence $\left(r_{\mathrm{s}}=-0.456\right.$; $p<0.05)$. Among the clinical data, we highlight the influence of previous treatment length in the improvement of the self-efficacy/adherence behaviours indices $\left(r_{\mathrm{s}}=\right.$ $0.385 ; p<0.05)$ and in the reduction of the perceived benefits indices $\left(r_{\mathrm{s}}=-0.438 ; p<0.05\right)$ (Table 5).

Predictors of effectiveness regarding the quality of life The greater the waist and hip circumferences, the better the results concerning multiple domains of weight-related quality of life (body esteem, social life and overall index of quality of life; $p<0.05)$. Mothers' higher occupational status was associated with an increased physical comfort $(\Delta M=19.91 \pm 22.67$ vs $\Delta M=0.84 \pm 10.82 ; U=23.000 ; p<0.05)$ and overall quality of life $(\Delta M=13.50 \pm 16.78$ vs $\Delta M=2.76 \pm 9.24 ; U=24.000$; $p<0.05)$. The adolescents who have benefited the most from the intervention program were the physically less active, those more sedentary and those who identified themselves with a bigger silhouette $(p<0.05)$. It was also found that the longer the previous treatment length, the greater the increase in body esteem $\left(r_{\mathrm{s}}=0.448 ; p<0.05\right)$. Furthermore, the adolescents who 
Table 2 Variation of lifestyle, adherence to weight control and quality of life during the 24 weeks and comparison of results between the control group and the experimental group (ITT and PP analysis)

\begin{tabular}{|c|c|c|c|c|c|c|c|}
\hline \multirow[t]{2}{*}{ Lifestyle } & \multicolumn{2}{|c|}{$\Delta(\mathrm{T} 2-\mathrm{T} 0)$} & \multicolumn{2}{|c|}{ Time effect } & \multicolumn{3}{|c|}{ Group effect } \\
\hline & $M$ & $\mathrm{SD}$ & $Z$ & $p$ & $\eta_{\mathrm{p}}^{2}$ & $\Lambda$ & $p$ \\
\hline \multicolumn{8}{|l|}{ Health responsibility } \\
\hline Next.Step-ITT & 0.15 & 0.46 & -1.858 & 0.063 & 0.027 & 0.763 & 0.382 \\
\hline Control & 0.003 & 0.43 & -0.328 & 0.743 & & & \\
\hline Next.Step-PP & 0.24 & 0.51 & -2.033 & 0.042 & 0.113 & 6.081 & 0.014 \\
\hline \multicolumn{8}{|l|}{ Physical activity } \\
\hline Next.Step-ITT & 0.14 & 0.49 & -2.242 & 0.025 & 0.160 & 2.388 & 0.122 \\
\hline Control & 0.12 & 0.61 & -1.112 & 0.266 & & & \\
\hline Next.Step-PP & 0.11 & 0.54 & -1.462 & 0.144 & 0.011 & 1.042 & 0.307 \\
\hline \multicolumn{8}{|l|}{ Nutrition } \\
\hline Next.Step-ITT & 0.09 & 0.33 & -1.903 & 0.057 & 0.000 & 0.066 & 0.797 \\
\hline Control & 0.09 & 0.29 & -1.756 & 0.079 & & & \\
\hline Next.Step-PP & 0.04 & 0.38 & -0.172 & 0.864 & 0.000 & 0.421 & 0.516 \\
\hline \multicolumn{8}{|l|}{ Positive life perspective } \\
\hline Next.Step-ITT & -0.03 & 0.49 & -0.707 & 0.480 & 0.002 & 1.861 & 0.172 \\
\hline Control & 0.05 & 0.36 & -0.963 & 0.336 & & & \\
\hline Next.Step-PP & -0.12 & 0.47 & -1.168 & 0.243 & 0.000 & 2.550 & 0.110 \\
\hline \multicolumn{8}{|l|}{ Interpersonal relations } \\
\hline Next.Step-ITT & 0.08 & 0.39 & -1.460 & 0.144 & 0.008 & 0.688 & 0.407 \\
\hline Control & 0.05 & 0.33 & -0.882 & 0.378 & & & \\
\hline Next.Step-PP & 0.16 & 0.40 & -1.837 & 0.066 & 0.052 & 2.772 & 0.096 \\
\hline \multicolumn{8}{|l|}{ Stress management } \\
\hline Next.Step-ITT & 0.05 & 0.40 & -0.473 & 0.636 & 0.000 & 0.000 & 0.995 \\
\hline Control & 0.06 & 0.40 & -1.118 & 0.264 & & & \\
\hline Next.Step-PP & 0.13 & 0.36 & -1.604 & 0.109 & 0.009 & 0.203 & 0.652 \\
\hline \multicolumn{8}{|l|}{ Spiritual health } \\
\hline Next.Step-ITT & 0.28 & 0.57 & -3.047 & 0.002 & 0.001 & 0.084 & 0.771 \\
\hline Control & 0.26 & 0.41 & -3.729 & 0.000 & & & \\
\hline Next.Step—PP & 0.27 & 0.64 & -1.868 & 0.062 & 0.000 & 0.002 & 0.962 \\
\hline \multicolumn{8}{|l|}{ ALP total score } \\
\hline Next.Step-ITT & 0.11 & 0.29 & -2.360 & 0.018 & 0.004 & 0.125 & 0.723 \\
\hline Control & 0.09 & 0.24 & -2.353 & 0.019 & & & \\
\hline Next.Step—PP & 0.12 & 0.32 & -1.615 & 0.106 & 0.029 & 2.098 & 0.147 \\
\hline \multicolumn{8}{|c|}{ Adherence to weight control } \\
\hline \multicolumn{8}{|c|}{ Risk of non-adherence to weight control } \\
\hline Next.Step-ITT & 0.02 & 0.71 & -0.299 & 0.765 & 0.002 & 1.239 & 0.266 \\
\hline Control & 0.10 & 0.65 & -0.710 & 0.478 & & & \\
\hline Next.Step-PP & 0.06 & 0.79 & -0.913 & 0.361 & 0.016 & 2.587 & 0.108 \\
\hline \multicolumn{8}{|c|}{ Self-efficacy and adherence behaviours } \\
\hline Next.Step—ITT & -0.10 & 0.76 & -1.399 & 0.162 & 0.001 & 0.101 & 0.750 \\
\hline Control & -0.14 & 0.80 & -1.327 & 0.184 & & & \\
\hline Next.Step-PP & 0.09 & 0.87 & -0.319 & 0.749 & 0.024 & 1.100 & 0.294 \\
\hline \multicolumn{8}{|c|}{ Parents and providers influence } \\
\hline Next.Step—ITT & -0.03 & 0.30 & -0.593 & 0.553 & 0.003 & 1.059 & 0.304 \\
\hline Control & -0.01 & 0.37 & -0.332 & 0.740 & & & \\
\hline Next.Step-PP & -0.06 & 0.31 & -1.030 & 0.303 & 0.010 & 3.731 & 0.053 \\
\hline
\end{tabular}


Table 2 (continued)

\begin{tabular}{|c|c|c|c|c|c|c|c|}
\hline \multirow[t]{2}{*}{ Lifestyle } & \multicolumn{2}{|c|}{$\Delta(\mathrm{T} 2-\mathrm{T} 0)$} & \multicolumn{2}{|c|}{ Time effect } & \multicolumn{3}{|c|}{ Group effect } \\
\hline & $M$ & SD & $Z$ & $p$ & $\eta_{p}^{2}$ & $\Lambda$ & $p$ \\
\hline \multicolumn{8}{|c|}{ Friends and school influence } \\
\hline Next.Step—ITT & -0.06 & 0.84 & -1.170 & 0.242 & 0.006 & 0.631 & 0.427 \\
\hline Control & -0.13 & 0.75 & -1.563 & 0.118 & & & \\
\hline Next.Step-PP & 0.21 & 0.99 & -0.767 & 0.443 & 0.051 & 2.624 & 0.105 \\
\hline \multicolumn{8}{|l|}{ Perceived benefits } \\
\hline Next.Step-ITT & -0.13 & 0.39 & -3.306 & 0.001 & 0.001 & 0.709 & 0.400 \\
\hline Control & -0.07 & 0.33 & -2.017 & 0.044 & & & \\
\hline Next.Step-PP & -0.24 & 0.29 & -3.465 & 0.001 & 0.025 & 1.373 & 0.241 \\
\hline \multicolumn{8}{|l|}{ TAWC total score } \\
\hline Next.Step-ITT & -0.08 & 0.44 & -1.979 & 0.048 & 0.004 & 0.560 & 0.454 \\
\hline Control & -0.10 & 0.41 & -1.665 & 0.096 & & & \\
\hline Next.Step-PP & 0.04 & 0.54 & -0.029 & 0.977 & 0.043 & 2.459 & 0.117 \\
\hline \multicolumn{8}{|l|}{ Quality of life } \\
\hline \multicolumn{8}{|l|}{ Physical comfort } \\
\hline Next.Step—ITT & 9.66 & 18.55 & -3.533 & 0.000 & 0.004 & 0.416 & 0.519 \\
\hline Control & 10.37 & 23.87 & -2.702 & 0.007 & & & \\
\hline Next.Step_PP & 10.77 & 19.12 & -2.782 & 0.005 & 0.009 & 0.012 & 0.912 \\
\hline \multicolumn{8}{|l|}{ Body esteem } \\
\hline Next.Step_-ITT & 7.92 & 22.22 & -2.092 & 0.036 & 0.020 & 0.133 & 0.716 \\
\hline Control & 15.36 & 21.00 & -3.911 & 0.000 & & & \\
\hline Next.Step-PP & 8.75 & 22.92 & -1.915 & 0.055 & 0.050 & 1.441 & 0.230 \\
\hline \multicolumn{8}{|l|}{ Social life } \\
\hline Next.Step—ITT & 7.23 & 15.17 & -3.203 & 0.001 & 0.014 & 3.770 & 0.052 \\
\hline Control & 11.19 & 18.08 & -4.108 & 0.000 & & & \\
\hline Next.Step-PP & 7.49 & 16.08 & -2.578 & 0.010 & 0.016 & 2.797 & 0.094 \\
\hline \multicolumn{8}{|l|}{ Family relations } \\
\hline Next.Step-ITT & 4.07 & 9.44 & -4.047 & 0.000 & 0.023 & 0.093 & 0.760 \\
\hline Control & 6.39 & 18.05 & -3.807 & 0.000 & & & \\
\hline Next.Step-PP & 3.09 & 6.31 & -2.545 & 0.011 & 0.047 & 0.239 & 0.625 \\
\hline \multicolumn{8}{|l|}{ IWQOL total score } \\
\hline Next.Step—ITT & 7.30 & 13.96 & -3.212 & 0.001 & 0.002 & 0.180 & 0.671 \\
\hline Control & 11.30 & 17.44 & -4.351 & 0.000 & & & \\
\hline Next.Step -PP & 7.66 & 14.00 & -2.799 & 0.005 & 0.021 & 0.309 & 0.578 \\
\hline
\end{tabular}

$A L P$ adolescent lifestyle profile, $T A W C$ treatment adherence to weight control, $I W Q O L$ Impact of Weight on Quality of Life, $P P$ per protocol analysis, ITT intention to treat analysis, $Z$ Wilcoxon-signed ranks test statistic, ${ }_{p}^{2}$ partial eta squared, likelihood ratio test statistic

did not mention previous attempts to control their weight showed better results in their social life $(\Delta M=18.32 \pm 24.64$ vs $\Delta M=3.77 \pm 8.20 ; U=21.000 ; p<0.05$ ) (Table 5).

\section{Discussion}

This study aimed to determine an e-therapeutic program's effectiveness when compared to a conventional treatment regarding the promotion of weight control, healthy lifestyles, adherence to weight control and quality of life.

The adolescents who joined the e-therapeutic program (PP analysis) showed an increase in the indices of health responsibility significantly higher than those from the control group. These differences correspond to a moderate effect size $(d=0.51)$ with clinical significance $[12,44]$. This positive effect of e-health interventions in the promotion of health responsibility is consistent with previous systematic reviews including children and adolescents $[4,26]$. 
Table 3 Variation of clinical outcomes at 24 weeks and comparison of results between the control group and the experimental group (ITT and PP analysis)

\begin{tabular}{|c|c|c|c|c|c|c|c|}
\hline \multirow[t]{2}{*}{ Clinical results } & \multicolumn{2}{|c|}{$\Delta(\mathrm{T} 2-\mathrm{T} 0)$} & \multicolumn{2}{|c|}{ Time effect } & \multicolumn{3}{|c|}{ Group effect } \\
\hline & $M$ & SD & $Z$ & $p$ & $\eta_{\mathrm{p}}^{2}$ & $\Lambda$ & $p$ \\
\hline \multicolumn{8}{|l|}{ BMI z-score (CDC) } \\
\hline Next.Step—ITT & -0.04 & 0.12 & -1.597 & 0.110 & 0.111 & 0.035 & 0.852 \\
\hline Control & -0.05 & 0.13 & -2.092 & 0.036 & & & \\
\hline Next.Step-PP & -0.04 & 0.10 & -1.737 & 0.082 & 0.006 & 0.122 & 0.727 \\
\hline \multicolumn{8}{|l|}{ BMI z-score (WHO) } \\
\hline Next.Step—ITT & -0.08 & 0.26 & -2.815 & 0.005 & 0.011 & 0.184 & 0.668 \\
\hline Control & -0.10 & 0.25 & -2.453 & 0.014 & & & \\
\hline Next.Step_PP & -0.11 & 0.19 & -2.557 & 0.011 & 0.001 & 0.422 & 0.516 \\
\hline \multicolumn{8}{|l|}{ Waist circumference } \\
\hline Next.Step—ITT & -1.28 & 2.20 & -4.367 & 0.000 & 0.014 & 0.022 & 0.882 \\
\hline Control & -1.80 & 1.51 & -5.267 & 0.000 & & & \\
\hline Next.Step_PP & -1.47 & 2.44 & -3.515 & 0.000 & 0.006 & 0.016 & 0.899 \\
\hline \multicolumn{8}{|l|}{ Hip circumference } \\
\hline Next.Step—ITT & 1.82 & 2.33 & -4.701 & 0.000 & 0.001 & 0.285 & 0.594 \\
\hline Control & 1.52 & 2.09 & -3.944 & 0.000 & & & \\
\hline Next.Step_PP & 2.09 & 2.87 & -3.243 & 0.001 & 0.001 & 1.051 & 0.305 \\
\hline \multicolumn{8}{|c|}{ Weekly physical activity } \\
\hline Next.Step_ITT & -0.42 & 1.89 & -1.435 & 0.151 & 0.001 & 0.091 & 0.763 \\
\hline Control & -0.67 & 1.62 & -3.018 & 0.003 & & & \\
\hline Next.Step-PP & -0.40 & 2.00 & -1.115 & 0.265 & 0.007 & 0.149 & 0.700 \\
\hline \multicolumn{8}{|l|}{ Screen time } \\
\hline Next.Step—ITT & -1.97 & 9.17 & -2.528 & 0.011 & 0.032 & 0.713 & 0.398 \\
\hline Control & -5.09 & 6.15 & -4.760 & 0.000 & & & \\
\hline Next.Step_PP & -2.30 & 7.77 & -2.330 & 0.020 & 0.010 & 0.590 & 0.442 \\
\hline \multicolumn{8}{|l|}{ Family support } \\
\hline Next.Step—ITT & -0.36 & 0.64 & -3.746 & 0.000 & 0.013 & 1.506 & 0.220 \\
\hline Control & -0.24 & 0.49 & -2.916 & 0.004 & & & \\
\hline Next.Step_PP & -0.18 & 0.61 & -1.945 & 0.052 & 0.004 & 0.170 & 0.680 \\
\hline \multicolumn{8}{|c|}{ Weight loss motivation } \\
\hline Next.Step—ITT & 0.09 & 0.82 & -0.975 & 0.329 & 0.023 & 0.489 & 0.484 \\
\hline Control & 0.39 & 0.65 & -3.813 & 0.000 & & & \\
\hline Next.Step_PP & 0.15 & 0.86 & -0.729 & 0.466 & 0.000 & 0.707 & 0.400 \\
\hline
\end{tabular}

ITT intention to treat analysis, $P P$ per protocol analysis, $Z$ Wilcoxon-signed ranks test statistic, ${ }_{\mathrm{p}}^{2}$ partial eta squared, likelihood ratio test statistic

Contrary to what has been described in previous studies [7], this program does not seem to have contributed to the improvement of adherence to weight control. Both the experimental and the control groups showed a significant decrease in the perceived benefits of adherence and a decrease in the overall index of adherence to weight control. These results seem to indicate that the adolescents felt it difficult to maintain high rates of treatment adherence, especially in cases of longterm treatments [37].

Regarding the program effectiveness on the weightrelated quality of life, significant differences between the two groups were not identified. Quality of life is not usually selected as one of the secondary outcomes of etherapeutic programs directed to children and adolescents, although previous studies with adults have also pointed out the presence of inconsistent results. While the study of Isabelle, Christelle, Patrick, Tony, Martial and Alain [19] found an improvement in the quality of life in obese patients undergoing a self-help program based on the Internet, other studies [22] did not show any additional benefit from the use of e-therapy in terms of quality of life compared to the usual treatment. 
Table 4 Analysis of predictors of effectiveness regarding the overweight and the lifestyle

\begin{tabular}{|c|c|c|c|c|c|c|c|c|c|c|}
\hline \multirow[t]{2}{*}{ Variables } & \multicolumn{2}{|c|}{$\Delta \mathrm{BMI}$ z-score } & \multicolumn{8}{|c|}{$\Delta$ lifestyle } \\
\hline & $\begin{array}{l}\mathrm{CDC} \\
\text { criteria }\end{array}$ & $\begin{array}{l}\text { WHO } \\
\text { criteria }\end{array}$ & $\Delta \mathrm{HR}$ & $\Delta \mathrm{PA}$ & $\Delta \mathrm{N}$ & $\Delta \mathrm{PLP}$ & $\Delta \mathrm{IR}$ & $\Delta \mathrm{SM}$ & $\Delta \mathrm{SH}$ & $\triangle \mathrm{ALP}$ \\
\hline \multicolumn{11}{|l|}{ Anthropometric data } \\
\hline Percentile of waist circumference $\left(r_{\mathrm{s}}\right)$ & 0.269 & 0.177 & 0.142 & 0.263 & 0.345 & -0.029 & 0.324 & 0.265 & 0.259 & 0.248 \\
\hline Hip circumference $\left(r_{\mathrm{s}}\right)$ & 0.140 & 0.084 & -0.195 & -0.063 & 0.331 & -0.073 & -0.032 & -0.020 & 0.226 & 0.015 \\
\hline \multicolumn{11}{|l|}{ Sociodemographic data } \\
\hline $\operatorname{Age}\left(r_{\mathrm{s}}\right)$ & 0.114 & 0.258 & -0.330 & -0.245 & -0.230 & -0.017 & -0.101 & -0.300 & -0.156 & -0.276 \\
\hline Gender $(U)$ & 74.000 & 71.500 & 59.500 & 69.500 & 61.500 & 57.500 & 53.500 & 62.000 & 60.500 & 65.000 \\
\hline Mother's academic qualification $(U)$ & 63.500 & 70.000 & 60.000 & 63.500 & 66.000 & 69.500 & 65.500 & 55.500 & 59.500 & 64.000 \\
\hline Father's academic qualification $(U)$ & 42.500 & 47.000 & 52.000 & 61.000 & 46.000 & 45.000 & 51.500 & 44.000 & 55.000 & 61.000 \\
\hline Mothers' occupational status $(U)$ & 39.500 & 42.000 & 49.500 & 50.500 & 44.500 & 50.500 & 54.000 & 37.000 & 38.500 & 53.000 \\
\hline Father's occupational status $(U)$ & 34.500 & 37.000 & 45.000 & $31.000 *$ & 59.000 & $26.500 *$ & 58.500 & 39.000 & 42.500 & 36.500 \\
\hline \multicolumn{11}{|l|}{ Behavioural data } \\
\hline Weekly physical activity $(\mathrm{h} / \mathrm{w})\left(r_{\mathrm{s}}\right)$ & 0.037 & 0.029 & $-0.471^{*}$ & $-0.556^{* *}$ & -0.320 & 0.048 & -0.336 & -0.065 & -0.207 & $-0.440 *$ \\
\hline Screen time $(\mathrm{h} / \mathrm{w})\left(r_{\mathrm{s}}\right)$ & 0.005 & -0.134 & -0.210 & 0.062 & $0.559 * *$ & -0.293 & 0.151 & 0.050 & -0.147 & 0.026 \\
\hline Family support $\left(r_{\mathrm{s}}\right)$ & -0.223 & -0.215 & -0.113 & -0.209 & 0.109 & -0.204 & 0.277 & -0.289 & -0.257 & -0.180 \\
\hline Weight loss motivation $\left(r_{\mathrm{s}}\right)$ & $-0.469^{*}$ & $-0.411^{*}$ & -0.222 & $-0.385^{*}$ & -0.100 & -0.270 & 0.104 & -0.235 & -0.268 & $-0.442 *$ \\
\hline Body image silhouette $\left(r_{\mathrm{s}}\right)$ & -0.137 & -0.143 & $0.440 *$ & 0.337 & 0.119 & 0.153 & 0.155 & $0.478^{*}$ & 0.191 & 0.255 \\
\hline \multicolumn{11}{|l|}{ Clinical data } \\
\hline Previous treatment length (months) $\left(r_{\mathrm{s}}\right)$ & 0.196 & 0.099 & 0.306 & 0.188 & 0.228 & 0.167 & -0.277 & 0.263 & 0.257 & 0.257 \\
\hline Age of onset of obesity $\left(r_{\mathrm{s}}\right)$ & -0.097 & -0.134 & -0.014 & -0.047 & 0.020 & -0.205 & -0.286 & 0.064 & $-0.562 * *$ & -0.257 \\
\hline Systolic blood pressure percentile $\left(r_{\mathrm{s}}\right)$ & 0.191 & 0.264 & 0.260 & 0.279 & -0.016 & 0.139 & 0.285 & 0.242 & 0.188 & 0.291 \\
\hline Diastolic blood pressure percentile $\left(r_{\mathrm{s}}\right)$ & 0.118 & 0.263 & 0.173 & 0.130 & -0.344 & 0.230 & 0.182 & 0.328 & 0.223 & 0.108 \\
\hline Binge eating episodes $(U)$ & 47.000 & 38.500 & 49.000 & 44.000 & 28.000 & 37.500 & 37.000 & 50.500 & 36.000 & 43.000 \\
\hline Previous attempts of weight loss $(U)$ & 28.000 & 25.000 & 31.000 & 35.000 & $10.500^{*}$ & 37.500 & 31.000 & 31.500 & 40.000 & 39.000 \\
\hline \multicolumn{11}{|l|}{ Data on the use of Next.Step } \\
\hline No. of accesses $\left(r_{\mathrm{s}}\right)$ & -0.204 & -0.185 & 0.110 & -0.093 & -0.111 & 0.221 & -0.110 & 0.134 & 0.077 & 0.032 \\
\hline Score obtained $\left(r_{\mathrm{s}}\right)$ & -0.296 & -0.313 & 0.067 & -0.101 & -0.011 & 0.207 & -0.025 & 0.168 & 0.123 & 0.022 \\
\hline
\end{tabular}

$H R$ health responsibility, $P A$ physical activity, $N$ nutrition, $P L P$ positive life perspective, $I R$ interpersonal relations, $S M$ stress management, $S H$ spiritual health, $A L P$ adolescent lifestyle profile, $U$ Mann-Whitney $U$ test statistic, $r_{s}$ Spearman correlation statistic

${ }^{*} p<0.05 ; * *<0.01$

According to our results, after 24 weeks of intervention, the experimental group participants showed a significant reduction of the BMI z-score, waist circumference and screen time $(p<0.05)$, although significant differences between groups were not found. Similar clinical results have been described in the literature $[4,26]$, although the empirical evidence of the effectiveness of virtual therapeutic communities is still fragile [16].

The lack of significant differences between the two groups might be related to the reduced time of intervention and implementation factors or may have resulted from the fact that the control group used a multidisciplinary intervention program instead of a "traditional" control group. Most probably, significant findings would be achieved if a control group without treatment had been used [42]. The option for this control group was based on ethical aspects and with the intention of minimizing the attrition rate, probably higher if the participants were allocated to a group without any kind of intervention. Moreover, we chose to use the e-therapy as an add-on and not as a substitute of the standard intervention, assuming the importance of the face-to-face contact with health professionals. A recent meta-analysis [29] which evaluated the impact of e-therapeutic interventions on weight reduction presented very relevant conclusions: the individuals who received the intervention as a complement to standard intervention lost significantly more weight, while the individuals for whom the intervention was a substitute of the traditional intervention lost significantly less weight. Baulch, Chester and Brennan [9] emphasize that although the modalities in-person may be preferred, the high prevalence rates of obesity suggest the urgency of expanding therapeutic options and 
Table 5 Analysis of predictors of effectiveness regarding the adherence to weight control and the quality of life

\begin{tabular}{|c|c|c|c|c|c|c|c|c|c|c|c|}
\hline \multirow[t]{2}{*}{ Variables } & \multicolumn{6}{|c|}{$\Delta$ adherence to weight control } & \multicolumn{5}{|c|}{$\Delta$ quality of life } \\
\hline & $\Delta$ SEA & $\Delta$ PPI & $\Delta \mathrm{FSI}$ & $\Delta \mathrm{PB}$ & $\begin{array}{l}\Delta \\
\text { TAWC }\end{array}$ & $\begin{array}{l}\Delta \\
\text { RNAWC }\end{array}$ & $\Delta \mathrm{PC}$ & $\Delta \mathrm{BE}$ & $\Delta \mathrm{SL}$ & $\Delta \mathrm{FR}$ & $\begin{array}{l}\Delta \\
\text { IWQOL }\end{array}$ \\
\hline \multicolumn{12}{|l|}{ Anthropometric data } \\
\hline $\begin{array}{l}\text { Percentile of waist circumference } \\
\left(r_{\mathrm{s}}\right)\end{array}$ & 0.046 & -0.105 & 0.183 & -0.165 & 0.072 & $-0.423^{*}$ & 0.266 & $0.512 * *$ & $0.595^{* *}$ & -0.086 & $0.498^{*}$ \\
\hline Hip circumference $\left(r_{\mathrm{s}}\right)$ & -0.037 & -0.146 & -0.037 & 0.054 & -0.133 & -0.184 & 0.337 & 0.327 & $0.432 *$ & -0.031 & $0.412 *$ \\
\hline \multicolumn{12}{|l|}{ Sociodemographic data } \\
\hline Age $\left(r_{\mathrm{s}}\right)$ & -0.302 & 0.163 & -0.206 & -0.172 & -0.278 & 0.098 & -0.062 & -0.252 & -0.173 & 0.324 & -0.185 \\
\hline Gender $(U)$ & 69.500 & 70.500 & 70.000 & 72.000 & 71.500 & 59.500 & 58.500 & 68.500 & 43.000 & 73.000 & 73.000 \\
\hline $\begin{array}{l}\text { Mother's academic qualification } \\
(U)\end{array}$ & 50.500 & 52.000 & 54.000 & $29.500^{*}$ & 68.000 & 63.500 & 53.500 & 49.000 & 52.000 & 51.000 & 46.000 \\
\hline $\begin{array}{l}\text { Father's academic qualification } \\
(U)\end{array}$ & 63.000 & 63.500 & 69.000 & 47.000 & 63.500 & 52.500 & 68.500 & 69.500 & 59.500 & 55.500 & 62.500 \\
\hline Mothers' occupational status $(U)$ & 43.000 & 44.500 & 53.000 & 34.500 & 52.000 & 38.500 & $23.000 *$ & 31.000 & 33.000 & 46.000 & $24.000 *$ \\
\hline Father's occupational status $(U)$ & 35.500 & 51.000 & 54.000 & 49.000 & 45.500 & 48.500 & 53.000 & 44.500 & 52.000 & 56.500 & 44.000 \\
\hline \multicolumn{12}{|l|}{ Behavioural data } \\
\hline $\begin{array}{l}\text { Weekly physical activity }(\mathrm{h} / \mathrm{w}) \\
\left(r_{\mathrm{s}}\right)\end{array}$ & -0.165 & -0.044 & -0.127 & -0.044 & -0.206 & 0.156 & -0.095 & -0.357 & -0.164 & -0.003 & $-0.406^{*}$ \\
\hline Screen time $(\mathrm{h} / \mathrm{w})\left(r_{\mathrm{s}}\right)$ & 0.011 & 0.034 & -0.122 & 0.302 & 0.039 & -0.057 & 0.161 & 0.292 & $0.499 *$ & 0.189 & $0.437 *$ \\
\hline Family support $\left(r_{\mathrm{s}}\right)$ & $-0.388^{*}$ & -0.209 & -0.269 & 0.202 & -0.368 & 0.146 & -0.057 & -0.301 & -0.037 & 0.027 & -0.255 \\
\hline Weight loss motivation $\left(r_{\mathrm{s}}\right)$ & -0.340 & -0.326 & $-0.449^{*}$ & 0.005 & $-0.456^{*}$ & 0.070 & -0.117 & -0.355 & -0.012 & 0.079 & -0.297 \\
\hline Body image silhouette $\left(r_{\mathrm{s}}\right)$ & $0.440^{*}$ & 0.011 & 0.248 & -0.300 & 0.370 & -0.071 & $0.442 *$ & $0.475^{*}$ & 0.233 & -0.155 & $0.412 *$ \\
\hline \multicolumn{12}{|l|}{ Clinical data } \\
\hline $\begin{array}{l}\text { Previous treatment length } \\
\text { (months) }\left(r_{\mathrm{s}}\right)\end{array}$ & $0.385^{*}$ & 0.011 & 0.255 & $-0.438^{*}$ & 0.324 & -0.074 & 0.090 & $0.448^{*}$ & 0.370 & -0.040 & 0.342 \\
\hline Age of onset of obesity $\left(r_{\mathrm{s}}\right)$ & -0.036 & 0.215 & -0.044 & 0.118 & 0.076 & 0.171 & 0.025 & 0.185 & -0.055 & 0.141 & 0.162 \\
\hline $\begin{array}{l}\text { Systolic blood pressure } \\
\text { percentile }\left(r_{\mathrm{s}}\right)\end{array}$ & 0.187 & 0.015 & 0.310 & -0.228 & 0.266 & -0.245 & 0.007 & 0.157 & -0.040 & -0.152 & 0.020 \\
\hline $\begin{array}{l}\text { Diastolic blood pressure } \\
\text { percentile }\left(r_{\mathrm{s}}\right)\end{array}$ & 0.138 & 0.040 & 0.370 & -0.116 & 0.238 & -0.303 & 0.060 & 0.106 & -0.089 & -0.248 & -0.018 \\
\hline Binge eating episodes $(U)$ & 39.500 & 44.000 & 40.000 & 50.500 & 49.000 & 47.000 & 29.500 & 36.500 & 49.500 & 42.000 & 28.500 \\
\hline $\begin{array}{l}\text { Previous attempts of weight loss } \\
(U)\end{array}$ & 43.000 & 41.000 & 38.000 & 40.000 & 44.000 & 43.000 & 43.000 & 32.500 & $21.000^{*}$ & 38.000 & 33.500 \\
\hline \multicolumn{12}{|l|}{ Data on the use of Next.Step } \\
\hline No. of accesses $\left(r_{\mathrm{s}}\right)$ & 0.198 & -0.104 & -0.042 & -0.040 & 0.057 & 0.129 & -0.219 & -0.134 & 0.036 & -0.313 & -0.285 \\
\hline Score obtained $\left(r_{\mathrm{s}}\right)$ & 0.183 & -0.077 & -0.052 & 0.140 & 0.062 & 0.080 & -0.095 & -0.029 & 0.201 & -0.350 & -0.109 \\
\hline
\end{tabular}

$R N A W C$ risk of non-adherence to weight control, $T A W C$ treatment adherence to weight control, $S E A$ self-efficacy and adherence behaviours, $P P I$ parents and providers influence, $F S I$ friends and school influence, $P B$ perceived benefits, $P C$ physical comfort, $B E$ body esteem, $S L$ social life, $F R$ family relations, IWQOL Impact of Weight on Quality of Life, $U$ Mann-Whitney $U$ test statistic, $r_{s}$ Spearman correlation statistic

$* p<0.05$

reducing the associated costs. These authors [9] believe that the Internet is increasingly recognized as an effective and innovative alternative as an add-on to face-to-face treatment programs. They further consider that the minimum contact with the health professional and the ability to disseminate individualized obesity management programs may increase the economic viability of programs for obesity based on the Internet.

It is possible that our finding of lack of improvement in the primary outcomes in the experimental group is due to limited exposure to the resources/materials relevant for the intervention, despite the fact that all participants enrolled in the experimental group have periodically received both automated messages and personalized messages sent through the platform. Being so, even if they did not access directly to the content of the platform, the motivational components were stimulated by receiving those feedback messages in their personal email.

The reduced adherence to e-therapeutic intervention programs for weight control in adolescents was already reported in previous studies [6], being considered as the main concern of this type of intervention [18]. A recent systematic review on 
the effect of e-therapeutic interventions for the treatment of obese adolescents [34] identified attrition rates between 4.9 and $30 \%$, while Weinstein's review [39] focusing on the adult population found attrition rates varying from 0 to $34 \%$. In the present study, the attrition rate was greater than the initial expectations (47.92\%), which suggests that the sample size may have been inadequate and that analyses may have been under-powered once the initial calculation of the sample size was based on an attrition rate of $30 \%$. Tershakovec and Kuppler [35] have already alerted to the dimension of the problem by stating that in their study about half of the obese children and adolescents under treatment dropped out. Furthermore, Next.Step participants are adolescents with severe overweight, reduced motivation and, in most cases, with a clinical past marked by several unsuccessful attempts of weight loss accumulated since a young age. These results underscore the importance of identifying ways to increase the adherence to this type of programs. A recent study offers an alternative to this problem, stating that adolescents tend to participate more consistently in an Internet-based program available during school hours rather than in a program designed to be carried out in their free time [25].

Baulch, Chester and Brennan [9] stress the importance of differentiating between the individuals more likely to benefit from an online program and those for whom the face-to-face treatment modalities are more appropriate. In this study, we found significant differences between the participants who have adhered and those who dropped out. The nonadherents were more sedentary, had a poorer diet and were less influenced by parents and providers in terms of adherence.

Direct intervention specifically for parents may be a relevant way to promote behavioural changes in the family environment [15]. Family support is crucial for children and adolescents and can positively influence their quality of life and their efforts for losing weight [40]. Families should be considered in the development of intervention programs [11]. In our study, parents were invited to join the program; however, they have not actively participated (attrition rate of $96.43 \%$ ). In future studies, it would be interesting to be able to increase the support and motivation provided by parents during the adolescent weight control program and then recalculate the program effectiveness [13].

A secondary objective of the study was the analysis of the predictors of the experimental program effectiveness. Reciprocal effects between the intervention outcomes were found, with several inter-correlations among the lifestyle, treatment adherence and quality of life dimensions. Previous studies have already suggested that weight reduction predicts improvement in the psychological domain [23] and in the health-related quality of life [20,36].

The initial motivation for weight loss was the strongest predictor for overweight reduction. Parental occupation, weekly physical activity, screen time, body image and presence of previous attempts of weight loss were predictors of effectiveness regarding lifestyle. Among the predictors of effectiveness regarding adherence to weight control were the longer treatment and mothers' higher academic education. A lower perception of the risk of non-adherence was associated with a higher waist circumference. The predictors of effectiveness concerning the quality of life were the waist and hip circumferences, the degree of mothers' education, the weekly amount of physical activity, screen time, the previous treatment length and previous attempts for weight loss. These findings are consistent with the results of previous studies $[24,38,45]$. It is recognized that parental involvement and the adolescent motivation for treatment are essential components for adherence to behavioural change [24]. We must underline the predictor effect of a higher maternal education in promoting treatment adherence, a fact that seems to be consistent with the fact of poverty and mother's low education being strongly associated with overweight [14, 41].

The results reported here should be considered in light of several strengths and limitations. Among the study limitations are the high attrition rate in the experimental group and the use of self-reported questionnaires. The answers provided may be biased by the social desirability, an aspect that we sought to be minimized by the guarantee of anonymity. Furthermore, the absence of random allocation of subjects to the experimental and control groups exposes this type of studies to a considerable number of threats regarding the internal and external validity, which may compromise the generalization of the results [17]. For this reason, both groups were initially evaluated concerning the homogeneity as a measure of control for the characteristics of the subjects. Despite that the adolescents of the control group have identified themselves with a body image silhouette slightly more obese, globally the two groups might be considered homogeneous and comparable [30]. However, we are aware that generalization of results might be done with caution. The absence of long-term follow-up to evaluate the maintenance of the behavioural change is an extra limitation of this study. This methodological option was due to the need to extend the recruitment sample and to the temporal constraints inherent to this research.

Among the strengths of this study, we underline its innovative character and contribution to the scientific knowledge in this field since it is one of the few contributions at the international level that evaluates an e-case management program specific for adolescents. Another aspect to highlight is the fact that the intervention program is supported by a multidisciplinary clinical team specialized in this field in a highly differentiated clinical setting. All the clinical parameters were assessed by the clinical team using objective anthropometric measures according to the standard international criteria (greater internal validity of the results). Another strength of this study is the use of obesity-specific instruments, more 
sensitive than generic ones, enabling to reduce the noise of medical co-morbidities [45]. Furthermore, it is important to mention the authors' option for evaluating the effectiveness of the program in detriment of its efficacy. The effectiveness analysis is a more pragmatic approach that seeks to analyse the results under conditions closer to real life, allowing for a greater heterogeneity in the sample. This strengthens the external validity of the study.

\section{Conclusions}

Despite having had a significant positive impact on multiple domains, the outcomes of this e-therapeutic program were not substantially different, in most of the studied variables, from those of the control group. These inconclusive results regarding this program's effectiveness compared to the standard multidisciplinary intervention give rise to an interesting discussion on cost-effectiveness. Further research is needed to be able to identify the potential benefits of a program of this kind in the clinical context. The identification of those benefits may facilitate future replication of the program at other institutions using the knowledge acquired with the strategies developed in here which involved a wide range of actors (adolescents, families and health professionals).

Acknowledgments This work was funded by Fundação para a Ciência e a Tecnologia (PTDC/DTP-PIC/0769/2012) and supported by the Polytechnic Institute of Leiria, Portugal, and the Department of Paediatrics of the at Hospital de Santa Maria, Lisbon, Portugal. We gratefully acknowledge the clinical staff of the Paediatric Obesity Clinic and the "Unidade de Ensino à Distância" for their dedication. We also thank all the adolescents and parents for their participation and collaboration.

\section{Conflict of interest None}

Author's contributions PS, HF, PG and FG were responsible for the study conception and design. PS and HF were responsible for data collection. PS and PG were responsible for the data analyses. All authors provided critical revision of all drafts of the manuscript, and read and approved the final manuscript.

\section{References}

1. Ahern D, Phalen J, Le L, Goldman R (2007) Childhood obesity prevention and reduction: role of eHealth. Health e-Technologies Initiative, Boston, Available at http://www.hetinitiative.org/ Childhood $\% 20$ Obesity $\% 20$ Prevention $\% 20$ and $\% 20$ Reduction $\%$ 20-\%20Role\%20of\%20eHealth.pdf

2. Alto Comissariado da Saúde [High Commissioner for Health] (2010) Plano Nacional de Saúde 2011-2016: Tecnologias de Informação e Comunicação. Available at: www.acs.min-saude.pt/pns2011-2016/ files/2010/07/TIC4.pdf Accessed 12 Feb 2012

3. American Psychological Association (2010) Ethical principles of psychologists and code of conduct. Am Psychol 65(5):493
4. An J, Hayman L, Park Y, Dusaj T, Ayres C (2009) Web-based weight management programs for children and adolescents: a systematic review of randomized controlled trial studies. Adv Nurs Sci 32:222-240

5. Baños RM, Cebolla A, Botella C, García-Palacios A, Oliver E, Zaragoza I, Alcaniz M (2011) Improving childhood obesity treatment using new technologies: the ETIOBE system. Clin Pract Epidemiol Ment Health 7:62-66

6. Baranowski T, Baranowski J, Cullen KW, Marsh T, Islam N, Zakeri I, Honess-Morreale L, deMoor C (2003) Squire's Quest! Dietary outcome evaluation of a multimedia game. Am J Prev Med 24:52-61. doi:10.1016/S0749-3797(02)00570-6

7. Battles HB, Wiener LS (2002) STARBRIGHT world: effects of an electronic network on the social environment of children with lifethreatening illnesses. Child Health Care 31:47-68. doi:10.1207/ S15326888CHC3101 4

8. Baulch J, Chester A, Brennan L (2008) Treatment alternatives for overweight and obesity: the role of online interventions. Behav Chang 25:1-14

9. Baulch J, Chester A, Brennan L (2010) Adolescent and parent content preferences and predictors of intention to use an online healthy weight website for adolescents. E-J Appl Psychol 6:18-26

10. Castelnuovo G, Manzoni GM, Cuzziol P, Cesa GL, Tuzzi C, Villa V, Liuzzi A, Petroni ML, Molinari E (2010) TECNOB: study design of a randomized controlled trial of a multidisciplinary telecare intervention for obese patients with type-2 diabetes. BMC Public Health 10: 204. doi:10.1186/1471-2458-10-204

11. Chen J-L, Weiss S, Heyman MB, Cooper B, Lustig R (2011) The efficacy of the web-based childhood obesity prevention program in Chinese American adolescents (Web ABC study). J Adolesc Health 49:148-154. doi:10.1016/j.jadohealth.2010.11.243

12. Cohen J (1988) Statistical power analysis for the behavioral sciences. L. Erlbaum Associates, Hillsdale

13. Doyle A, Goldschmidt A, Huang C, Winzelberg A, Taylor, Wilfley D (2008) Reduction of overweight and eating disorder symptoms via the Internet in adolescents: a randomized controlled trial. J Adolesc Heal 43:172-179. doi:10.1016/j.jadohealth.2008.01.011

14. Drewnowski A (2004) Obesity and the food environment: dietary energy density and diet costs. Am J Prev Med 27:154-162. doi:10. 1016/j.amepre.2004.06.011

15. Epstein LH (1996) Family-based behavioural intervention for obese children. Int J Obes Relat Metab Disord 20(Suppl 1):S14-S21

16. Eysenbach G, Powell J, Englesakis M, Rizo C, Stern A (2004) Health related virtual communities and electronic support groups: systematic review of the effects of online peer to peer interactions. BMJ 328: 1166. doi:10.1136/bmj.328.7449.1166

17. Fortin M (2009) Fundamentos e etapas do processo de investigação. Lusociência, Loures

18. Glasgow RE, Nelson C, Kearney KA, Reid R, Ritzwoller DP, Strecher VJ, Couper MP, Green B, Wildenhaus K (2007) Reach, engagement, and retention in an Internet-based weight loss program in a multi-site randomized controlled trial. J Med Internet Res 9:e11. doi:10.2196/jmir.9.2.e11.PMCID

19. Isabelle C, Christelle C, Patrick R, Tony L, Martial V, Alain G (2011) Acceptance and efficacy of a guided Internet self-help treatment program for obese patients with binge eating disorder. Clin Pract Epidemiol Ment Health 7:8-18

20. Kolotkin RL, Meter K, Williams GR (2001) Quality of life and obesity. Obes Rev 2:219-229. doi:10.1111/j.1467-789X.2001.00040.x

21. Kuczmarski RJ, Ogden CL, Grummer-Strawn LM (2000) CDC growth charts: United States. Adv Data 1-27

22. McConnon A, Kirk SF, Cockroft JE, Harvey EL, Greenwood DC, Thomas JD, Ransley JK, Bojke L (2007) The Internet for weight control in an obese sample: results of a randomised controlled trial. BMC Health Serv Res 7:206. doi:10.1186/1472-6963-7-206 
23. Myers MD, Raynor HA, Epstein LH (1998) Predictors of child psychological changes during family-based treatment for obesity. Arch Pediatr Adolesc Med 152:855-861

24. Naar-King S, Jen K (2011) Interventionist procedures for adherence to weight loss. Recommendations in black adolescents. Available at http://clinicaltrials.gov/ct2/show/NCT01350531

25. Neil AL, Batterham P, Christensen H, Bennett K, Griffiths K (2009) Predictors of adherence by adolescents to a cognitive behavior therapy website in school and community-based settings. J Med Internet Res 11:e6. doi:10.2196/jmir.1050

26. Nguyen B, Kornman K, Baur L (2011) A review of electronic interventions for prevention and treatment of overweight and obesity in young people. Obes Rev 12:e298-e314. doi:10.1111/j.1467-789X. 2010.00830.x

27. Onis M, Onyango AW, Borghi E, Siyam A, Nishida C, Siekmann J (2007) Development of a WHO growth reference for school-aged children and adolescents. Bull World Health Organ 85:660-667. doi:10.2471/BLT.07.043497

28. Palmeira A, Martins S, Costa R, Quaresma A, Fonseca H, Teixeira P, Kolotkin R (2008) Confirmatory factor analysis of the Portuguese version of the Impact of Weight on Quality of Life - Kids. Obesity 16(S1):S112

29. Reed V, Schifferdecker K, Rezaee M, O’Connor S, Larson R (2011) The effect of computers for weight loss: a systematic review and meta-analysis of randomized trials. J Gen Intern Med 27(1):99-108

30. Sampieri RH, Collado CF, Lucio PB (2006) Metodologia da Pesquisa. McGraw-Hill, São Paulo

31. Sousa P, Fonseca H, Gaspar P (2013) Measuring adherence to weight control in adolescents: development and validation of a self-report questionnaire. Aten Primaria 45(Sup I):70-71

32. Sousa P, Gaspar P, Fonseca H, Hendricks C (2013) Health promoting behaviours in adolescence: psychometric properties of the adolescent lifestyle profile PT version. Aten Primaria 45(Sup I):140

33. Sousa P, Fonseca H, Gaspar P, Gaspar F (2013) Internet-based intervention programme for obese adolescents and their families (Next.Step): research protocol of a controlled trial. J Adv Nurs. doi: 10.1111/jan.12245
34. Sousa (2012, in press) Efetividade dos programas de intervenção de e-saúde em adolescentes obesos: revisão sistemática da literatura. Revista Pensar Enfermagem

35. Tershakovec AM, Kuppler K (2003) Ethnicity, insurance type, and follow-up in a pediatric weight management program. Obes Res 11: 17-20. doi:10.1038/oby.2003.4

36. Tsiros MD, Olds T, Buckley JD, Grimshaw P, Brennan L, Walkley J, Hills AP, Howe PR, Coates AM (2009) Health-related quality of life in obese children and adolescents. Int J Obes (Lond) 33:387-400. doi:10.1038/ijo.2009.42

37. Vieira M, Atihê R, Soares R, Damiani D, Setian N (2004) Initial compliance of obese adolescents in weight-reduction programs. Pediatria 26(4):240-246

38. Wadden T, West D, Neiberg R, Wing RR, Ryan DH, Johnson KC, Foreyt JP, Hill JO, Trence DL, Vitolins MZ (2009) One-year weight losses in the Look AHEAD study: factors associated with success. Obesity 17:713-722

39. Weinstein PK (2006) A review of weight loss programs delivered via the Internet. J Cardiovasc Nurs 21:251-258

40. White M, Martin PD, Newton RL, Walden HM, York-Crowe EE, Gordon ST, Ryan DH, Williamson DA (2004) Mediators of weight loss in a family-based intervention presented over the internet. Obes Res 12:1050-1059. doi:10.1038/oby.2004.132

41. WHO (2007) The challenge of obesity in the WHO European Region and the strategies for response. World Health Organization, Geneva

42. Wieland L, Falzon L, Sciamanna C, Trudeau K, Brodney S, Schwartz J, Davidson K (2012) Interactive computer-based interventions for weight loss or weight maintenance in overweight or obese people (review). Cochrane Database Syst Rev 8, CD007675

43. WMA General Assembly (2008) World Medical Association Declaration of Helsinki: ethical principles for medical research involving human subjects (as amended by the 59th WMA General Assembly, Seoul, October 2008). World Med Assoc

44. Wolf (1986) Meta-analysis: quantitative methods for research synthesis. Sage, Beverly Hills

45. Zeller M, Modi A (2008) Psychosocial factors related to obesity in children and adolescents. In: Jelalian E, Steele R (eds) Handbook of childhood and adolescent obesity. Springer, New York, pp 25-42 